\title{
Study of Fuzzy Logic-based Controller for Diff-Serv Bandwidth Broking
}

\author{
Hadj Bourdoucen ${ }^{1,2}$, Fatma Al-Azani ${ }^{2}$ and Ahmed Al-Naamany ${ }^{3}$ \\ ${ }^{1}$ Communication and Information Research Center (CIRC), Sultan Qaboos University, Oman \\ 2 Department of Electrical and Computer Engineering, Sultan Qaboos University, Oman \\ ${ }^{3}$ Modern College of Business and Science, Sultan Qaboos University, Oman
}

\begin{abstract}
Combining transmission of both voice and data on the same network infrastructure was a challenging task which required an advanced network architecture which needs to be scalable and simple. This resulted in a new approach for Ethernet systems where Differentiated Service (Diff-Serv) was introduced to respond to this need and in addition, improve Quality of service (QoS) as a way of providing class of services for different end users. Therefore, optimizing the use of the network available bandwidth is a prim goal of this work which is focused on investigating the impact of implementing a fuzzy logic controller of the crucial factors affecting the performance of the network. These external or internal factors will be controlled by the developed Fuzzy Logic system that will act as bandwidth broker (BB) to provide each user with his optimal Code Point (CP). Within the scope of this work, the CP will not only involve packet loss rate as an external factor to check the network congestion, but in addition, it will consider the internal factors; which are a combination of both service-level agreement (SLA) and the type of user application used, as external factors as well. The Code Point will be marked in the transmitted packets, where it will be possible to the router to check it, and will treat it as per the agreement between the user and the administrator.
\end{abstract}

Keywords: fuzzy logic controller, diff-serv, service-level agreement, SLA, QoS, bandwidth broker, code point

\section{Introduction}

The huge growth of the businesses and the increasing societal needs lead to develop more internet technologies that depend on real-time applications which come over the capabilities of available network resources. The lack of reaching expected high performance is a real concern that needs to be well addressed by researchers.
Today's network communication performance buzzwords include mainly: latency, jitter, bandwidth and packet loss. In addition, differentiation service is also a serious issue that needs to be looked at since the current datagram strategy is dealing with all transmitted and received packets in the same way, thus, offering a single level of service. Therefore, real time applications under this type of service became impracticable.

Without Quality of service (QoS) capabilities, it is impossible for a service provider to offer service differentiation to customers, where these expect that services should be linked to the cost paid for. This is a very important reason for QoS, and indeed, it forms one of the bases by which traffic congestion can be managed. In line with this, it is important to work symbiotically with service providers to differentiate product offerings for different customers. This means that QoS for a network might be segmented into a number of performance levels, with different access priced at each level (A. H. Aghvami et al. 2004, S. Chen et al. 2000, F.-C. Ren at al. 2002, O. Aboul-Magd and B. Jamoussi 2001, http: www.qosforum. com 1999.).

Among the two most popular basic types of QoS available are the resource reservation (integrated services) and prioritization (differentiated services). These measurable services have to be provided for different types of traffic, particularly those in real-time. The services can 
be implemented in different forms, but the simplest and most scalable approach for Ethernet is Differentiated Services (Diff-Serv).

Bandwidth prioritization is one solution for providing network QoS. For this research work, a number of papers have been published focusing mainly on two key factors: type of application and bandwidth availability (e.g. L. Yunyue and W. Qishi 2013, C. C. Chollette et al. 2012, and the references therein). In this line, another research work has considered one more factor in addition to these two, to generate the differentiated services code points (DSCP) and treat the packets in a more optimal way (A. S. Al-Belushi 2006). The work was based on developing a bandwidth controller based on Fuzzy logic that gives each user his optimal Code Point (CP) by considering his class, type of application and available bandwidth. In this case, the router checks each transmitted packet's CP and treats it as contracted. A method to classify users and priorities to share transmission rate through a typical WAN link was suggested. The approach was to develop and further investigate the impact of essential factors on performance of the fuzzy logic controller (Z. Wang 2001, A. Krishnamurthy et al. 2005, G. Stattenberger and T. Braun 1999). The present work focuses mainly on considering more factors influencing the bandwidth prioritization that limits the QoS. Such factors include: delay (jitter), throughput, key protocols, and computation overhead.

After studying the fuzzy logic controller model which acts as a bandwidth broker (A. Sohail and S. Khanum 2008, M. Goel et al. 2004, I. Okumus et al. 2003, Y.-D. Lin et al. 2001, R. Zhang et al. 2000) and examination of different applications of fuzzy logic approaches for designing decision algorithms for queue scheduling, mobility management in heterogeneous wireless network and self-tuning fuzzy controller for maximum drop probability management (C. C. Chollette et al. 2012, Y. Tao, R. Peng, 2011, H. Wang et al. 2009) the resulting Diff-Serv QoS is explored accordingly.

Therefore, this work will describe a Fuzzy logic based controller that gives each user the optimal code point, taking into account the above mentioned factors which are Packet Loss (PL), Round Trip Time (RTT), application, SLA and BW.

\section{Design and Set-up of Fuzzy Logic Controller}

The first attempt in the design of Fuzzy logic controller was to present all the five factors together as variables and study the relationship between them. As a result, the following two options have been considered:

- Option 1: Build a single Fuzzy logic controller which deals with all the five factors as inputs that result in one output code point which will be marked on the packets. A trial has been done to use a FL toolbox to build a controller that has all these five factors together at the same time. This option was judged to produce a complex controller as it will have around $2^{32}$ possibilities to be investigated. This is not simple, neither practical to implement through this tool.

- Option 2: Create two separate Fuzzy logic controllers FL1 and FL2 and multiplex them. In this case, two FL parts need to be presented separately. For instance, the main five rules used are presented in Table 1, whereas in Table 5, only three parameters out these five are indicated (Bandwidth, SLA and Packet loss). It is worth noting that in the first part, Table 1 was simplified to present only the most effective rules of the controller. This option has been implemented in the simulator and the results will be presented in the coming sections.

With regard to the first part of option 2, the membership function for the inputs and code point of Fuzzy Logic controller 1 (FL1) are shown in Figure 1 a through d. For the SLA, three membership functions have been introduced which are low, mid and high. The SLA has five classes that vary from one to five all over the research work, where the preferred user was assigned to class five.

The second factor presented here is the available bandwidth (BW) which is assumed to be varying from 0 to $100 \%$. The BW membership functions are considered to be Low, Mid and High as shown in Figure 1b. However, the application type which is the third key factor was assumed to be varying from 0 to 1 where pure real-time applications are presented by a value 


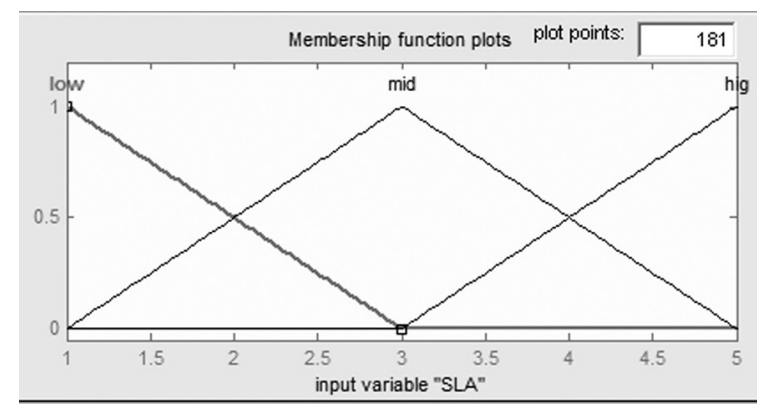

(a)

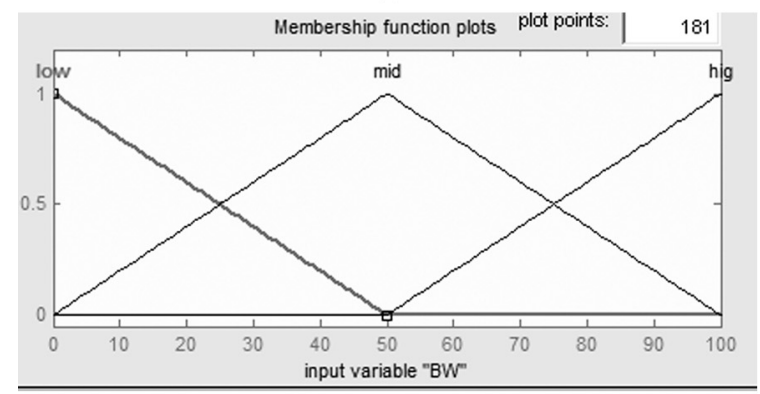

(b)

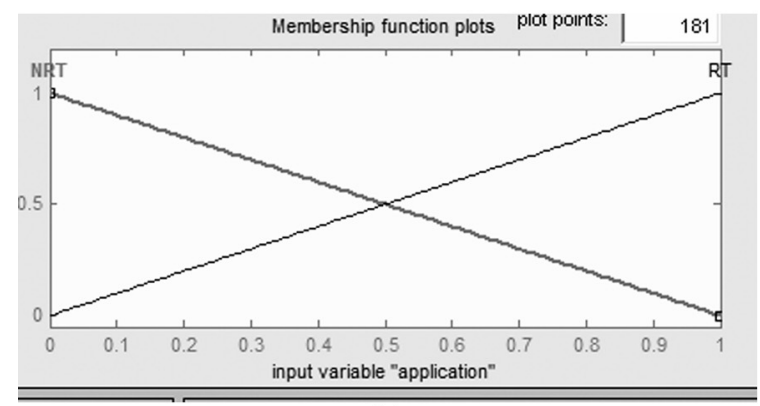

(c)

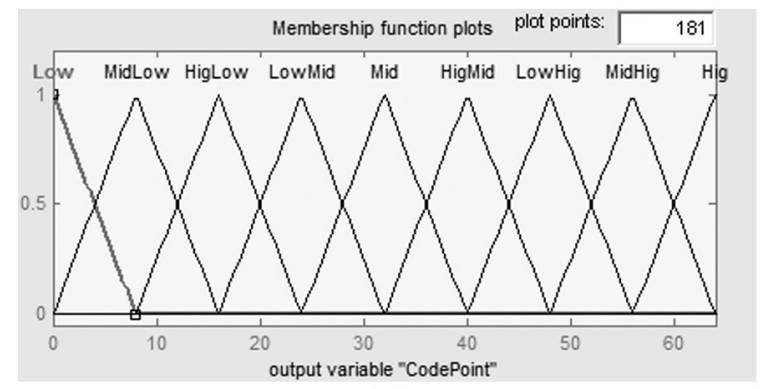

(d)

Figure 1. Fuzzy Logic membership functions of controller FL1.

1 , while the non-real time applications were presented by value 0 . The corresponding membership functions are NRT and RT (respectively for non-real time and real time applications).

The membership functions applied on this FL controller are shown in Figure 1d. There are nine functions used which are: Low, MidLow, HigLow, LowMid, Mid, HigMid, LowHig, MidHig and Hig, which are varying on a scale from 0 to 64 . This adopted scale can be well justi-

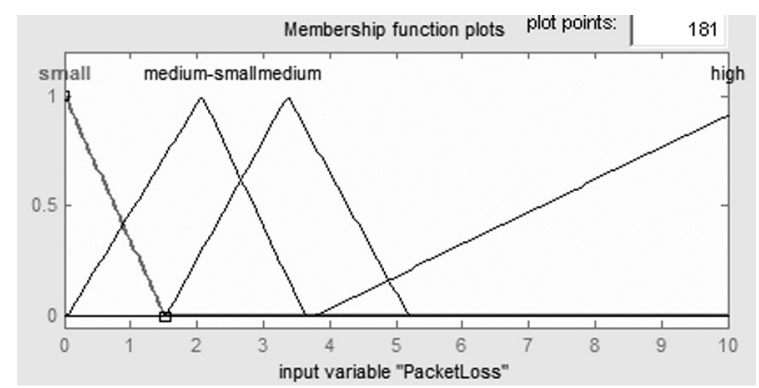

(a)

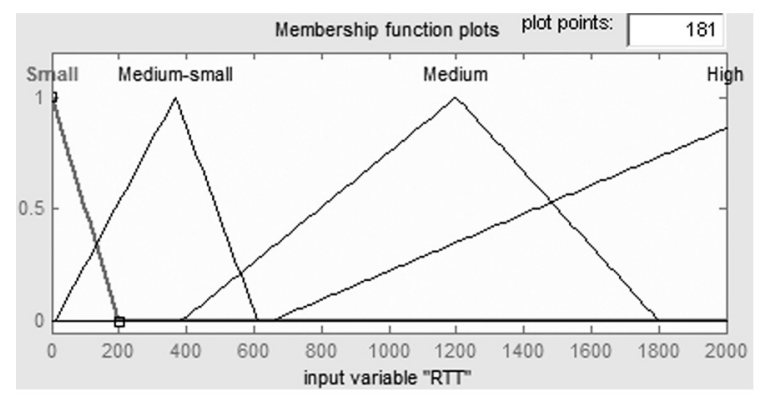

(b)

Figure 2. Fuzzy Logic membership functions of controller FL2.

fied since the Diff-Serv byte overrides existing definition of IPv4 TOS byte (Type of Service byte) and IPv6 traffic class octet. Note that six bits are used as Diff-Serv code point (DSCP) to select the Per-Hop-Behavior (PHB), a packet experience at each node where the other two bits are currently unused.

This work deals with a complete Diff-Serv scheme with detailed access node function, in which there are totally 64 levels of services for both real time and non-real time flows.

At Fuzzy Logic 2 controller (FL2), two factors have been considered which are the Packet Loss rate (PL) and the Round Trip Time (RTT). In fact, four membership functions of the PL were designated in order to give more highlight and differentiation. These are shown in Figure 2 a and $b$ which are Small, Medium-small, Medium and High.

On the other hand, four membership functions were also considered for the RRT, which are Small, Medium-small, Medium and High as shown in Figure 2b.

To perform numerical simulations, the two developed controllers FL1 and FL2 have been combined in a single Matlab Simulink model as shown in Figure 3 where a summation function was used in the simulation code. Some of the obtained results from simulation analysis are depicted in Figure 4. 


\begin{tabular}{|c|c|c|c|c|c|c|c|}
\hline \multicolumn{2}{|c|}{ Fuzzy Logic 1 factors } & \multicolumn{3}{|c|}{ Fuzzy Logic 2 factors } & \multirow{2}{*}{ FL1 Decision } & \multirow{2}{*}{ FL2 Decision } & \multirow{2}{*}{ FL Decision } \\
\hline RTT & $\mathrm{PL}$ & Application & SLA & $\mathrm{BW}$ & & & \\
\hline Small & Small & $\overline{\mathrm{NR}}$ & Low & Low & $\begin{array}{l}\text { Positive } \\
\end{array}$ & 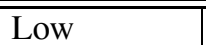 & 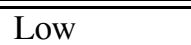 \\
\hline Small & Med small & NR & Low & Med & $\begin{array}{l}\text { Positive } \\
\text { small }\end{array}$ & Mid low & Mid low \\
\hline Small & Med & NR & Low & High & Zero & High low & High \\
\hline Small & High & NR & Mid & Low & Neg small & Low mid & Low \\
\hline Med small & Small & NR & Mid & Med & $\begin{array}{l}\text { Positive } \\
\text { small }\end{array}$ & Mid & Med \\
\hline Med small & Med small & NR & Mid & High & Zero & High mid & High \\
\hline Med small & Med & NR & High & Low & Neg small & Low high & Med \\
\hline Med small & High & NR & High & Med & Neg med & Med high & Low \\
\hline Med & Small & NR & High & High & Zero & High & High \\
\hline Med & Med small & $\mathrm{R}$ & Low & Low & Neg small & Low & Low \\
\hline Med & Med & $\mathrm{R}$ & Low & Med & Neg med & Low & Med \\
\hline Med & High & $\mathrm{R}$ & Low & High & $\mathrm{Neg}$ & Low & Low \\
\hline High & Small & $\mathrm{R}$ & Mid & Low & Neg small & Low mid & Mid low \\
\hline High & Med small & $\mathrm{R}$ & Mid & Med & Neg med & Mid & Med \\
\hline High & Med & $\mathrm{R}$ & Mid & High & $\mathrm{Neg}$ & High mid & Med \\
\hline High & High & $\mathrm{R}$ & High & Low & $\mathrm{Neg}$ & High & Low \\
\hline
\end{tabular}

Table 1. The five factors presented in one FL system with their relationships.

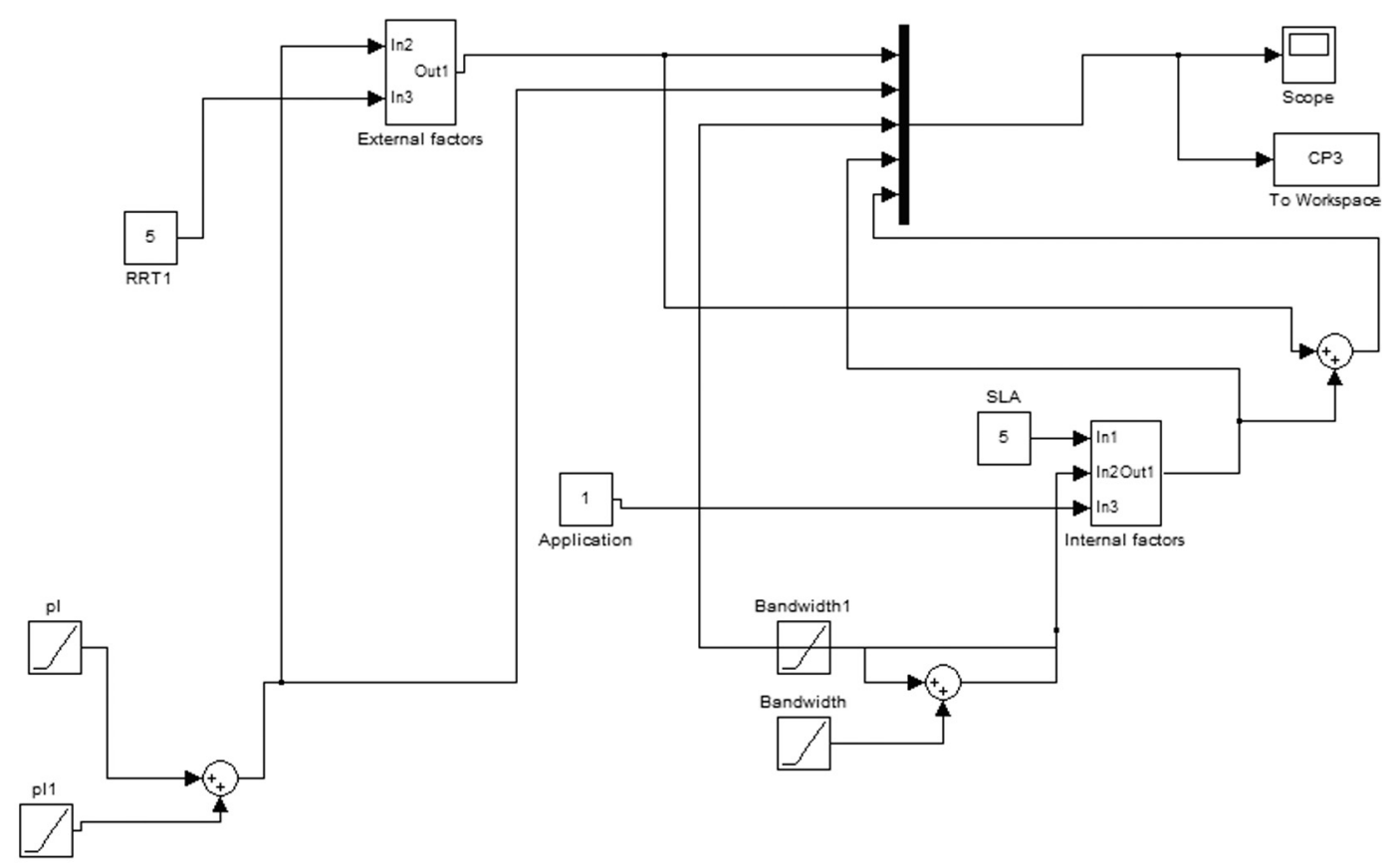

Figure 3. Simulation model for combined FL1 and FL2 controllers.

Note that from examination of the obtained simulation results shown in Figure 4, the Code Point obtained from the product of the two controllers does not give a clear picture of the treatment of the data. Therefore, using the summation function at this level will not give a fair representation to this relationship with regard to these factors as a whole. 


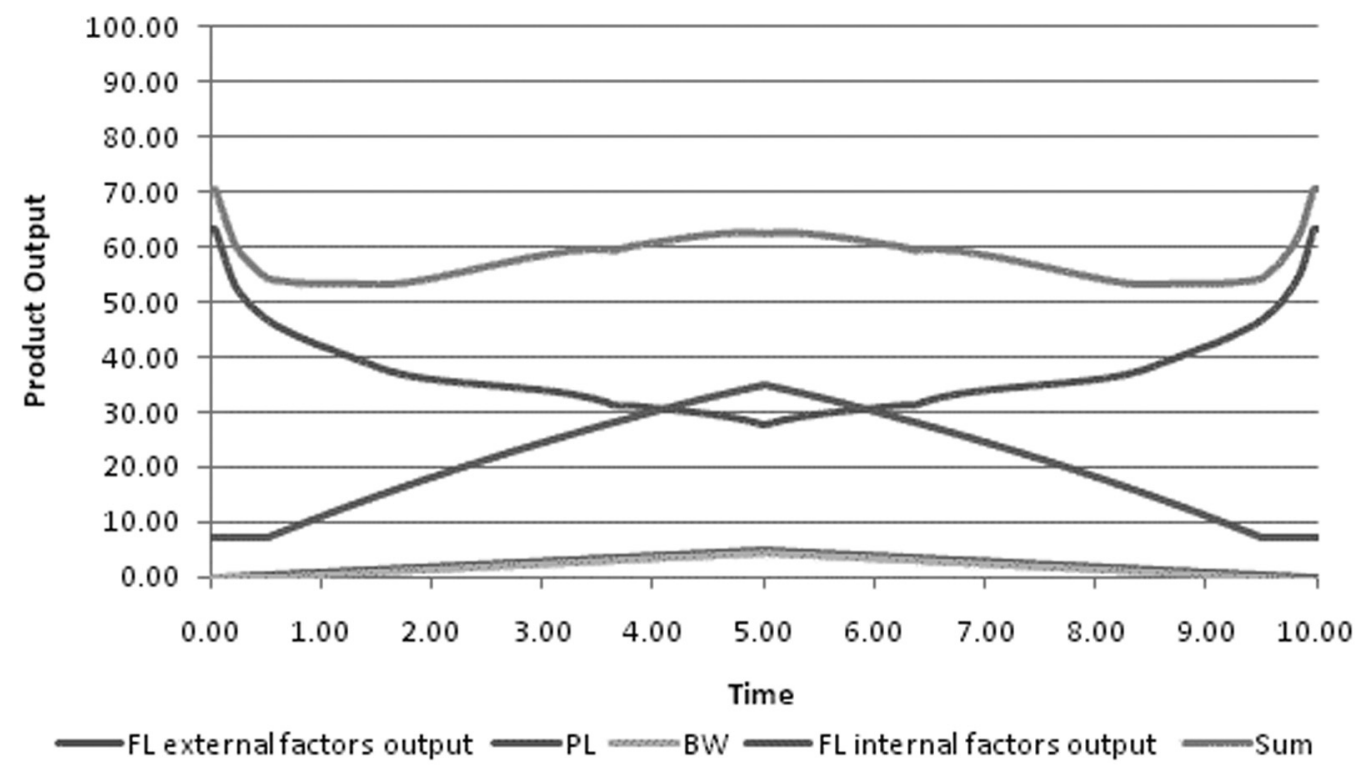

Figure 4. Results product of FL1 and FL2.

It has been noticed that the most effected factors in the controller can be reduced to three factors only. These are, the Packet Loss from the first fuzzy logic controller, the Bandwidth and the SLA from the second fuzzy logic controller. As a result of this assumption, a second trial with the new factors has been introduced.

Therefore, in the second attempt, the number of factors is reduced to three which are expected to have more effects on the final results. Thus, we ended up with BW, SLA and Packet Loss. Table 5 shows all the rules controlling the FL at this stage.

In this scenario, the SLA was presented as a matrix for both the "user class" that is varying between Low, Med and High and the "application type" that can be "non-real time", "other" and "real time" application as shown in Tables 2,3 and 4.

This will give, as pointed out earlier, more highlights and differentiation in the obtained results.

For different types of applications which might start with sensitive voice traffic up to email and/or games, the users can be classified into five classes where the priority will be given to class 5 to be served first, i.e. before the rest of the classes.

\begin{tabular}{|c||l|l|}
\hline & Application Type & Representation Number \\
\hline \hline \multirow{2}{*}{ RT } & Video conferencing & 1 \\
\cline { 2 - 3 } & Voice over IP & 1 \\
\hline \multirow{4}{*}{ Other } & GCC & 0.8 \\
\cline { 2 - 3 } & FTP & 0.5 \\
\cline { 2 - 3 } & Database & 0.2 \\
\cline { 2 - 3 } & WWW & 0.1 \\
\hline \multirow{3}{*}{ NRT } & PDF file & 0 \\
\cline { 2 - 3 } & Email and chat & 0 \\
\cline { 2 - 3 } & Gaming & 0 \\
\hline
\end{tabular}

Table 2. Application Type.

\begin{tabular}{|l||l|}
\hline Class \# & Probability of drop \\
\hline Class 1 & Higher \\
\hline Class 2 & "" \\
\hline Class 3 & Middle \\
\hline Class 4 & "" \\
\hline Class 5 & Lower \\
\hline
\end{tabular}

Table 3. SLA probability of drop.

\begin{tabular}{|l|l|l|l|}
\hline & NRT & Other & RT \\
\hline High & 3 & 4 & 5 \\
\hline Medium & 2 & 3 & 4 \\
\hline Low & 1 & 2 & 3 \\
\hline
\end{tabular}

Table 4. SLA Matrix. 


\begin{tabular}{|c|c|c|c|c|}
\hline \multicolumn{5}{|c|}{ Packet Loss $=$ Low } \\
\hline & & \multicolumn{3}{|l|}{ BW } \\
\hline & & Low & Mid & High \\
\hline \multirow{3}{*}{ SLA } & Low & Low Mid & Mid & High Mid \\
\hline & Mid & Mid & High Mid & High \\
\hline & High & High Mid & High & VHigh \\
\hline \multicolumn{5}{|c|}{ Packet Loss = Mid } \\
\hline & & \multicolumn{3}{|l|}{ BW } \\
\hline & & Low & Mid & High \\
\hline \multirow{3}{*}{ SLA } & Low & Low & Low Mid & Mid \\
\hline & Mid & Low Mid & Mid & High Mid \\
\hline & High & Mid & High Mid & High \\
\hline \multicolumn{5}{|c|}{ Packet Loss $=$ High } \\
\hline & & \multicolumn{3}{|l|}{ BW } \\
\hline & & Low & Mid & High \\
\hline \multirow{3}{*}{ SLA } & Low & VLow & Low & Low Mid \\
\hline & Mid & Low & Low Mid & Mid \\
\hline & High & Low Mid & Mid & High Mid \\
\hline
\end{tabular}

Table 5. Fuzzy Logic rules between Bandwidth, SLA and Packet loss.

As shown in Table 5, there are three main factors that were considered in this part which have major effects on the results of simulation.

The membership function for the fuzzy inputs and code points for the combined FL are shown in Figure $5 \mathrm{a}-\mathrm{d}$ respectively. All the factors of membership functions used above were reutilized as they were (e.g. the packet loss doesn't have any changes and can be pulled from the edge router through running a script on the bandwidth broker (BB) server to perform its function).

It is worth noting that the SLA in the above case has five classes, but with presentations different from the previous case, since they resulted from the combination of both the application type and administration classification of the user. The same applies to the available bandwidth where the BB will read its status from the edge router. Note also that the rules used for this case are extracted from Table 5 and configured at the fuzzy logic tool box as shown in the section below. The range of the code points varies between 0 and 64. At the edge router, for instance, if the code point equals to $\mathrm{N}$, it means that $\mathrm{N}$ out of 64 were passed forward and the others were dropped.

\subsection{Processing a request}

The developed model in this work is expected to be connected to a core switch of a network where processing capabilities are expected to be very high, as represented in Figure 6 . The model can be run as a separate program within a server which can communicate to the database server (DB). The database can run in both oracle or SQL database and might carry any network and $\mathrm{AD}$ information. The routers connected to ingress/egress router to ISP and the links between access routers and clients/servers are of $1 \mathrm{Gbps}$ Ethernet.

The following steps explain various envisaged communication phases involved to process any request from the user.

1. A user sends a message request that has all details about his data e.g. user name, password, etc...

2. FL controller validates the user name, password, and the class by checking the database.

3. Database sends back the answer for validation and class number.

4. FLC requests the edge router about the bandwidth details and the percentage of packet loss. 
5. Edge router sends back the percentage of both available bandwidth and packet loss.

6. FLC sends the generated code point to the user.

7. The user configures the given code point which will be placed in packets' headers.

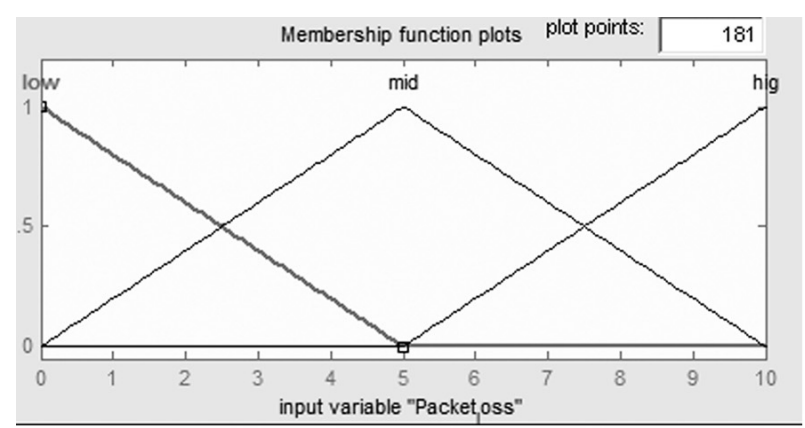

(a)

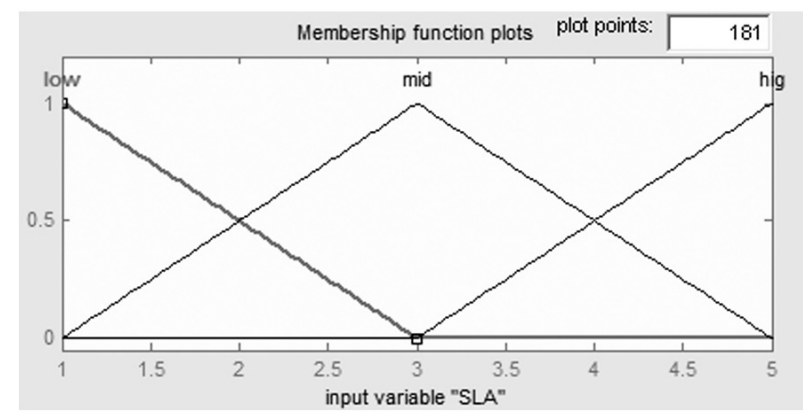

(b)

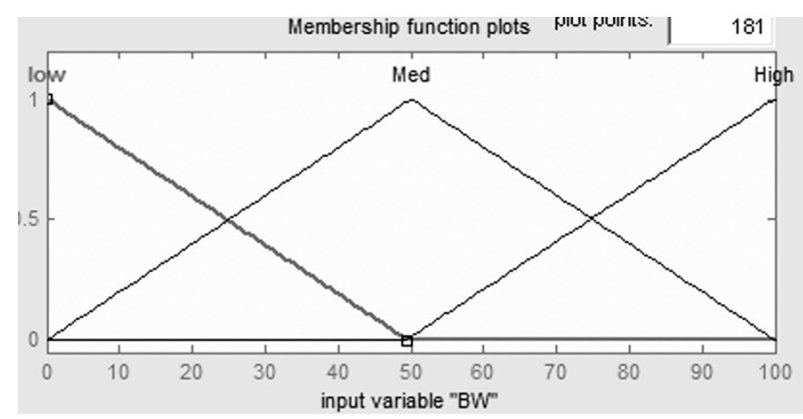

(c)

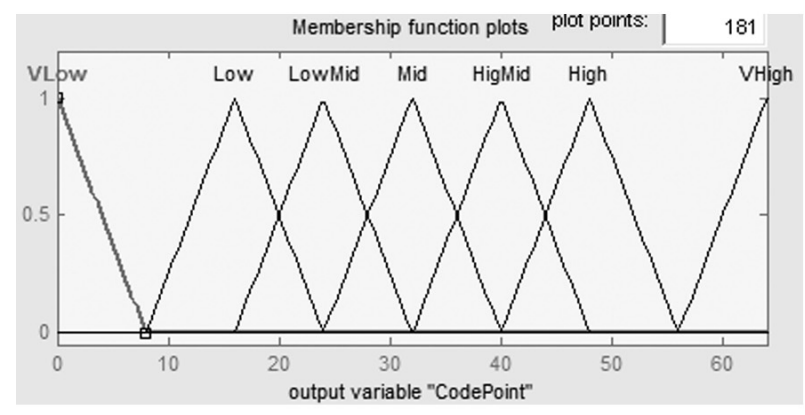

(d)

Figure 5. Fuzzy Logic membership functions combined FL.

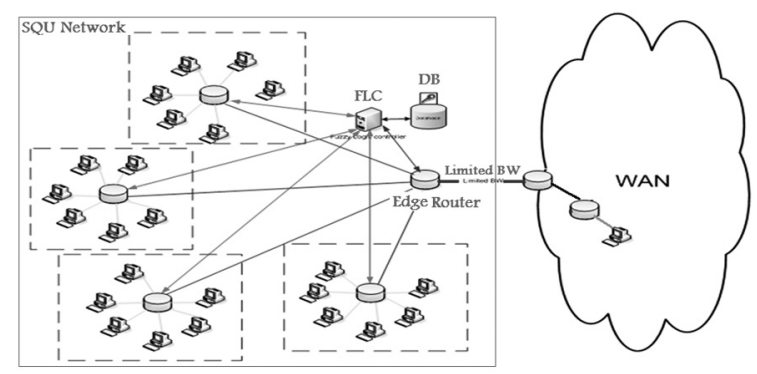

Figure 6. Comprehensive system network.

\subsection{Model implementation and development in Matlab}

In order to implement the whole system and improve the FL controllers presented in previous works, additional important factors have been considered to enhance the performance of the suggested model. Matlab's fuzzy logic toolbox was used to implement the developed FL system where its Simulink layout model is shown in Figure 7.

Comparison of the output Code Points for different values of SLA varying from 1 to 5 for the developed model was done.

\section{Analysis and Results}

To demonstrate the validity of the developed approach, a set of representative results obtained from the Simulink model are shown in Figures 8 through 10.

The results for a time range of 200, where the Bandwidth is changing from 0 to its peak value of 100 and then back to zero, are as shown in Figure 8.

This figure also shows that the packet loss reaches its highest value, which corresponds to $\mathrm{PL}=10$, and in this case there will be no much differentiation between users since the available bandwidth is very low.

However, when the packet loss reaches its middle rate, the bandwidth broker will start showing much more difference for users having different SLA values (refer to Figure 9). 


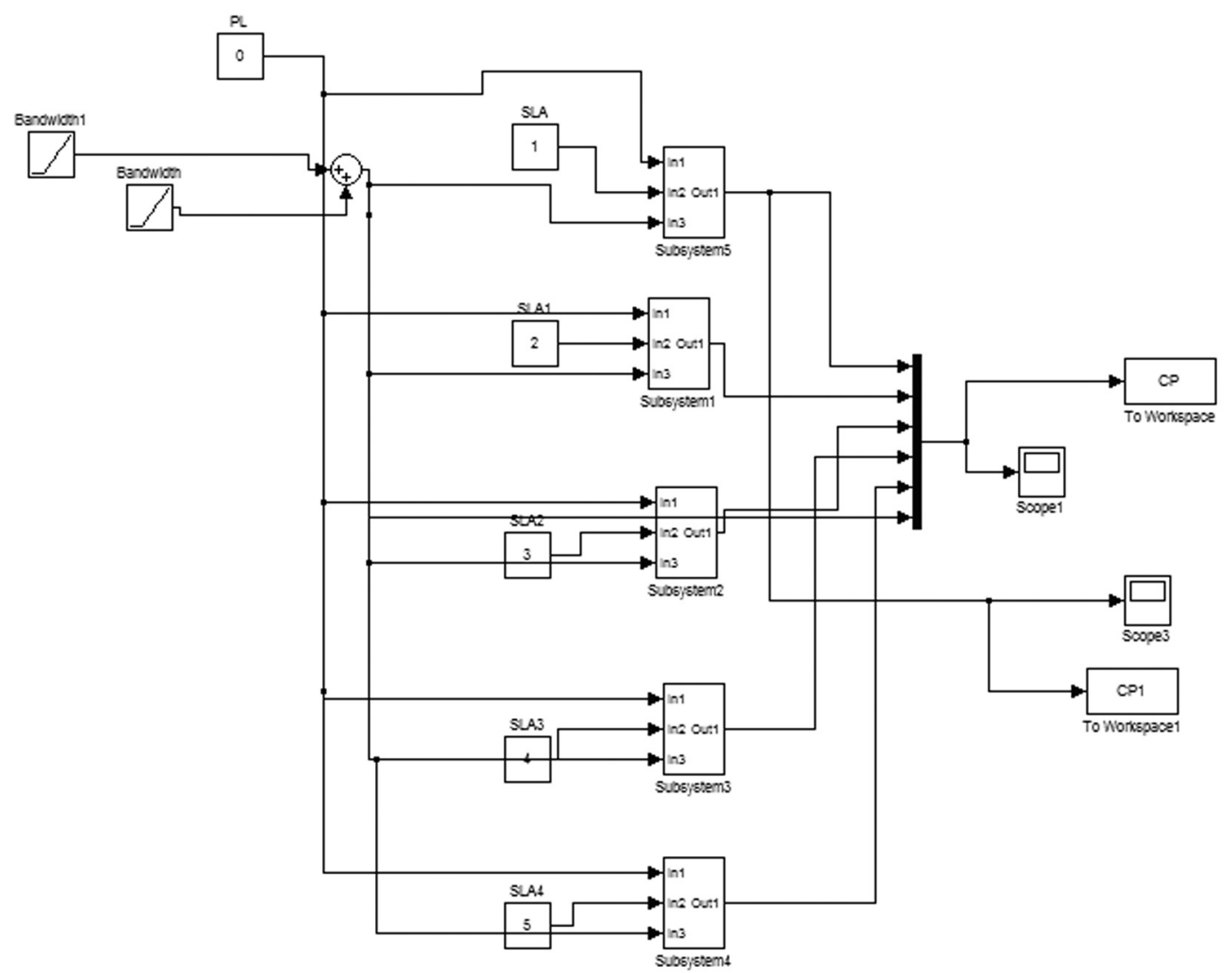

Figure 7. Matlab Simulink window of the developed model layout.

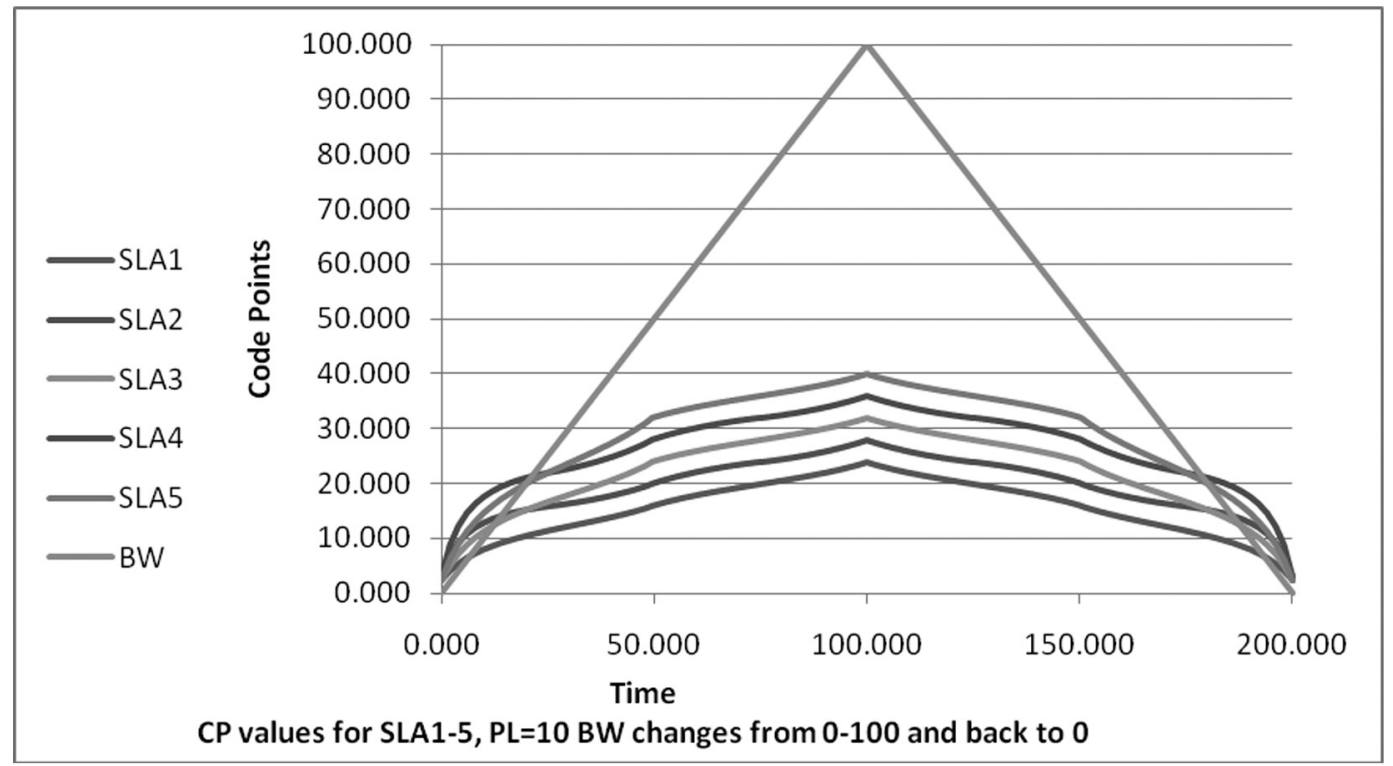

Figure 8. $\mathrm{CP}$ values for $\mathrm{SLA}=1-5, \mathrm{PL}=10$ and $\mathrm{BW}$ changes from $0-100$ and then back to 0 . 


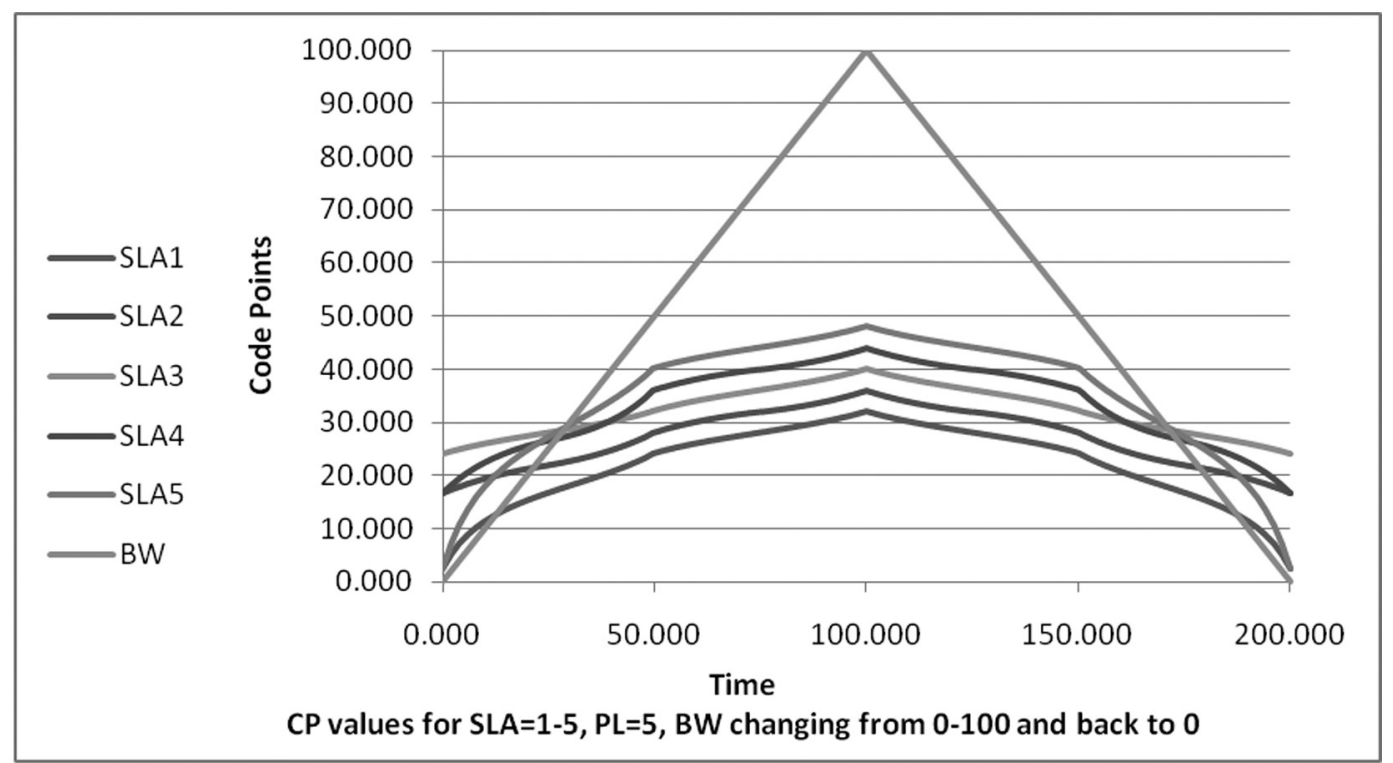

Figure 9. $\mathrm{CP}$ values for $\mathrm{SLA}=1-5, \mathrm{PL}=5$ and $\mathrm{BW}$ changes from $0-100$ and then back to 0 .

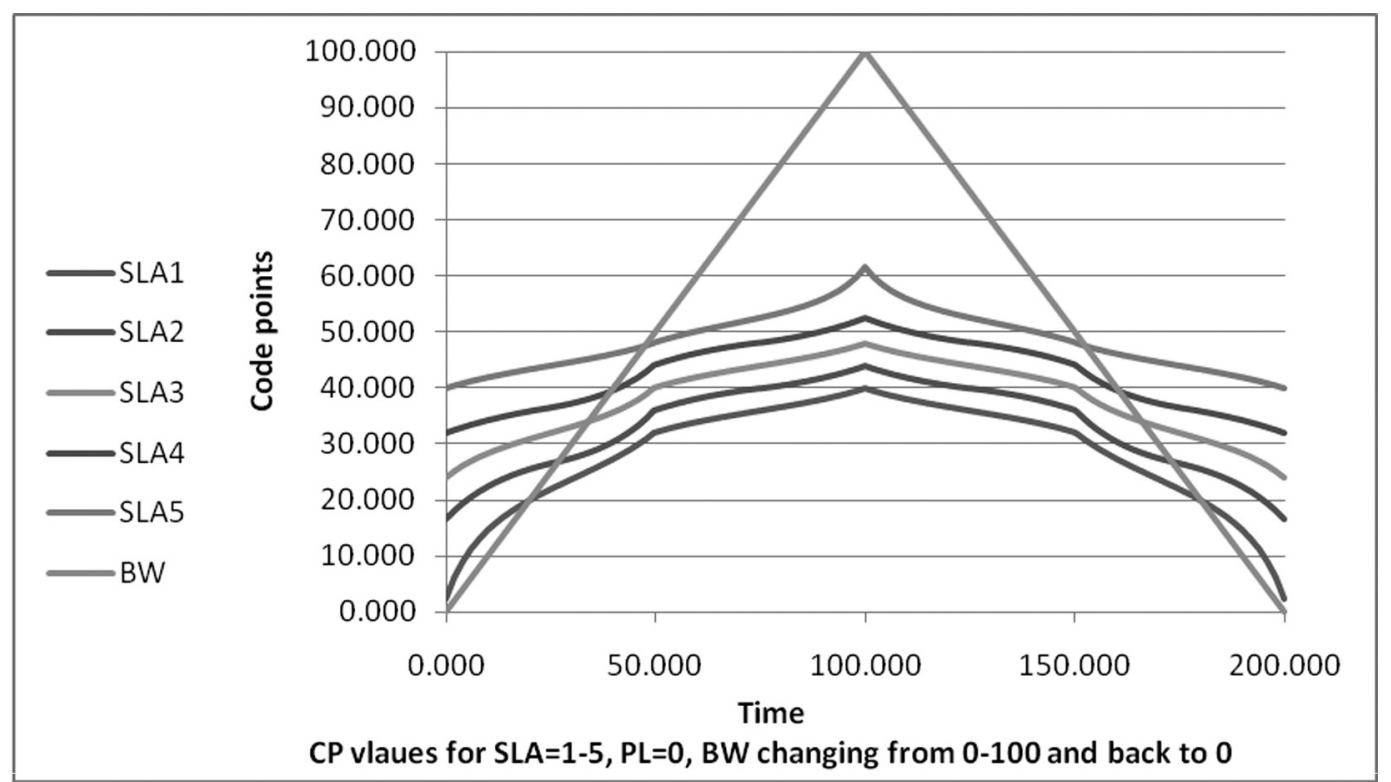

Figure 10. $\mathrm{CP}$ values for $\mathrm{SLA}=1-5, \mathrm{PL}=0$ and $\mathrm{BW}$ changes from $0-100$ and back to 0 .

On a stage where the packet loss is zero, the fuzzy logic bandwidth broker will try from first moment to provide the priority to the user with highest SLA as shown in Figure 10.

\section{Conclusion}

Quality of Service is considered as one way to provide a class of service with consistency, predictability and data delivery which will ensure customers' satisfaction with the service provided. Diff-Serv as a technology is one solution to provide QoS in the network. In this work, the controlling factors were classified as external and internal factors, including Packet Loss, SLA and available bandwidth. The SLA is taken as a matrix of class of the user specified by the network administrator and the application type.

The optimal policy from the architecture of the fuzzy logic controller was determined where the scaling factors for all fuzzy logic inputs were 
determined first. The SLA matrix output was set to 5 levels in the simulation. The bandwidth percentages were selected to be more general instead of using an exact amount since they differ from one network to another and the packet loss was varying from 0 to 10 . Using both external and internal factors, it was possible to determine the decision code point via fuzzification, fuzzy interference and de-fuzzification.

It was noticed from the results that if the packet loss is zero, which means no network congestion outside the network, and the SLA is 5 with $50 \%$ of bandwidth available; the $\mathrm{CP}$ grade can reach up to 47 . If the packet loss is 5 , and the SLA is also 5 with $50 \%$ of bandwidth available, the CP can go down to reach 39 for its best. If the packet loss reaches its maximum value which is 10 , and the SLA reads 5 with the same available bandwidth $50 \%$, the $\mathrm{CP}$ grade will be 32. It was clear from this work how the controller is rewarding each user, depending on the mentioned factors. Thus, to achieve high performance communication networks that satisfy the business current needs, a simple and easily administrable network control mechanism is required.

\section{References}

[1] A. Krishnamurthy, L. Qian, Y. Wang, P. DAUCHY, A. CONTE, A new coordination scheduling algorithm in distributed bandwidth broker QoS architecture. Dept. of Computer. Science, Texas Univ., Richardson, TX, USA. Aug, 2005.

[2] C. C. Chollette, A. K. U. Chude-OKonkwo, K. A. BAKAR, O. V. MATTHEWS, A queue scheduling approach to quality of service support in Diff-Serv networks using fuzzy logic. International Conference on Computer and Communication Engineering (ICCCE), 2012.

[3] F.-C. Ren, C.-J. Chang, R.-G. Cheng, QoSguaranteed fuzzy transmission controller for dynamic TDMA protocol in multimedia communication systems. IEE Proceedings - Communications, vol. 149, no. 56, 2002.

[4] G. Stattenberger, T. Braun, Performance of Bandwidth Broker for Diff-Serv Networks. Institute of Computer Science and Applied Mathematics, University of Bern, Neubrückstr. 10, 3012 Bern, Switzerland. Jan, 1999.

[5] H. Aghvami, A. Ganz, R. SivaKumar, H. Zheng, W. ZHU, C.-T. LEA, Advanced Mobility Management and QoS Protocols for Next-Generation Wireless Internet. IEEE Journal on Selected Areas in Communications, vol. 22, no. 5, 2004.
[6] H. Wang, Z. Ji, Y. Sun, A Fuzzy Logic Based Congestion Control Algorithm Suitable for IP-Based Broadband Satellite Networks, Sixth International Conference on Fuzzy Systems and Knowledge Discovery, FSKD '09, vol. 4, 2009.

[7] L. YunYUE, W. QISHI, Complexity Analysis and Algorithm Design for Advance Bandwidth Scheduling in Dedicated Networks, tIEEE/ACM Transactions on Networking, vol. 21, 1, 2013.

[8] M. Goel, M. Moh, T. Hasegawa, S. Ano, Differentiated-service enabled group communications using bandwidth brokers. Performance, Computing, and Communications Conference, 2004.

[9] O. Aboul-Magd, B. Jamoussi, QoS and service interworking using constraint route label distribution protocol (CR-LDP). IEEE Communications Magazine, vol. 39, no. 5, 2001.

[10] Okumus, S. J. Chapin, H. Junseok, H. MaNTAR, Scalability of inter-domain edge tunnels using bandwidth brokers: a region-based approach. Global Telecommunications Conference, GLOBECOM '03. vol. 5, 2003.

[11] QOS PROTOCOLS \& ARCHITECTURES, 1999, http: wWw. qosforum. com

[12] R. Zhang, J. Ma, Fuzzy QoS management in DiffServ networks. International Conference on Systems, Man, and Cybernetics, vol. 5, 2000.

[13] S. AL-BELUSHI, Bandwidth Prioritization Provisioning for Inter-router Networks. MSc thesis report, Department of Electrical and Computer Engineering, SQU, Sultanate of Oman, February, 2006.

[14] S. Chen, K. NAhrstedt, Y. ShavitT, A QoS-aware multicast routing protocol. IEEE Journal on Selected Areas in Communications, vol. 18, no. 12, 2000.

[15] Sohail, S. Khanum, Simplifying Network Management with Fuzzy Logic. Dept. of computer. Eng., NUST, Rawalpindi, May, 2008.

[16] Y. TAO, R. Peng, A fuzzy logic vertical handoff algorithm with motion trend decision. $6^{\text {th }}$ International Forum on Strategic Technology (IFOST), vol. 2, 2011.

[17] Y.-D. Lin, C.-H. Chang, Y.-C. HsU, Bandwidth brokers of instantaneous and book-ahead requests for differentiated services networks. Global Telecommunications Conference, GLOBECOM '01. vol. 4, 2001.

[18] Z. WANG, Internet QoS: Architectures and Mechanisms for Quality of Services. Morgan Kaufmann Publishers, San Francisco, CA, March, 2001. 
Received: December, 2012

Revised: April, 2013

Accepted: May, 2013

Contact addresses:

Hadj Bourdoucen Communication and Information Research Center ECE Dept., College of Engineering Sultan Qaboos University PO Box 33

Al Khod, 123 Muscat

Oman

e-mail: hadj@squ.edu.om

Fatma A. S. Al-Azani ECE Dept., College of Engineering Sultan Qaboos University

PO Box 33

Al Khod, 123 Muscat

Oman

e-mail: f.azani@hotmail.com Ahmed Al-Naamany
Modern College of Business \& Science PO Box 100

Al-Khuwair, 133

Sultanate of Oman e-mail: naamany@mcbs.edu.om

HADJ BOURDOUCEN obtained his PhD degree from Ecole Centrale de Lyon, France, in 1987. Worked at the University of Science and Technology Houari Boumedienne (USTHB), Algeria, 1987-90, then at the National Institute of Electricity and Electronics (INELEC), Boumerdes, 1990-97. He joined Sultan Qaboos University (SQU) in 1998 where he served as Head of Department of Information Engineering, then as Head of Department of Electrical and Computer Engineering until 2007. He is presently a Professor at ECE Dept., and Director of Communication and Information Research Center (CIRC), SQU. His research activities and interests include communication \& networking, modelling, simulation \& performance evaluation, integrated electronics and hardware design. In these areas he published over 100 research publications and edited several books and conference proceedings. Dr. Bourdoucen is an IEEE Senior member.

FATMA AL-AZANI obtained her Bachelor degree from Sultan Qaboos University, Oman, in June 2003 and the Master degree from the same university in 2010. She worked in Oil field industry at Petroleum Development Oman (PDO) from 2004 to 2008 as a network engineer. She has been working in Occidental of Oman Inc. as a Telecom and network specialist for around five years. Her research activity for the Master degree was about bandwidth prioritization using customized Fuzzy logic model. The model functions to check three factors to decide the best treatment for the received data in order to utilize the bandwidth provided by the ISP when traffic sent through the internet. The three factors were the user class, RT/ Non-RT applications, and the bandwidth available at the time of the request. The scenario built into an environment such as university where there are different user levels of e.g. management, university faculty and students.

AhMed Al-NaAmany is Associate Professor at Sultan Qaboos University. He obtained his Ph.D. from the University of Manchester Institute of Science and Technology, UK, 1995, M.Sc. (EE-Computer Control) from Drexel University, United States, 1990, B.Sc. (MultiDisciplinary Engineering.) from Widener University, 1986, B.Sc. (EE) from Widener University 1986 (With Honors). He is a member of IEEE Computer and Control Societies. He has lectured on computer architecture, computer control, computer networks and artificial intelligence applications. Dr. Al-Naamany is also a recipient of Fulbright Scholarship from USA, 1999. Currently, he is the Dean of Modern College of Business \& Science, and Chairman of IEEE, Oman section. 
